\title{
Quantum-Inspired Neural Networks with Application
}

\author{
Jianping Li \\ School of Computer and Information Technology, Northeast Petroleum University, Daqing, China \\ Email: leejp@126.com
}

Received 13 May 2015; accepted 24 May 2015; published 27 May 2015

Copyright (C) 2015 by author and Scientific Research Publishing Inc.

This work is licensed under the Creative Commons Attribution International License (CC BY). http://creativecommons.org/licenses/by/4.0/

c. (i) Open Access

\begin{abstract}
In this paper, a novel neural network is proposed based on quantum rotation gate and controlledNOT gate. Both the input layer and the hide layer are quantum-inspired neurons. The input is given by qubits, and the output is the probability of qubit in the state $|1\rangle$. By employing the gradient descent method, a training algorithm is introduced. The experimental results show that this model is superior to the common BP networks.
\end{abstract}

Keywords

Quantum Computing, Neural Networks, Algorithm Design

\section{Introduction}

In the eighties of the twentieth century, Benioff and Feynman proposed the concept of quantum computation, and then P.W. Shor gave the first quantum algorithm of very large integer factorization [1] in 1994 and L.K. Grover proposed a quantum algorithm which searches a marked state in an unordered list [2] in 1996. Quantum computation has been widely paid attention and become a challenging research line. Fuzzy logic, evolution calculation, and neural networks are regarded as the most promising three important aspects in the artificial intelligence field, which compose intelligence calculation (soft calculation) and have much comparability with quantum computation. Therefore, the combination of them would bring promising research in theory.

Some research results in the nineties of the twentieth century show that [3]-[5] the information processing of brain may be related to the quantum states; there may exists the effect of quantum mechanics in brain, and the quantum system has the same dynamics features as the biology neural networks. Fatt and Katz proved that in biology neuron, the neurotransmitter is released from the nerve terminals by the form of multi-molecule and small units, and they call this kind of small units quantum. Each of small units (quantum) describes the minimal unit that the neurotransmitter is released. In general, although the number of quantum in each synaptic response of 
neurons may be different, the number of molecules in each quantum is the same. Therefore, the combination of quantum computation and ANN may preferably simulate the information processing of brain.

However, as yet, there is little understanding of the essential components of artificial neural networks based on quantum theoretical concepts and techniques. The basal model and theory of quantum neural networks are in research. At present, there is not a set of perfect theory to direct the construction of model. Since Kak firstly proposed the concept of quantum neural computation [6] in 1995, the quantum neural networks have been paid attention, and many novel ideas and elementary model have been proposed. In 1997, N. B. Karayiannis et al. proposed the model of quantum neural networks with multilevel hidden neurons based on the superposition of quantum states in quantum theory. ZHU Da-qi et al. applied this model to fault diagnosis for photovoltaic radar electronic equipment, and acquired the satisfied results [7]. In 2000, Matsui et al. proposed a quantum neural networks model based on the single bit rotation gate and the 2-bit controlled-NOT gate, presented the algorithm of model, and investigated its performance on solving the 4-bit parity check problem and the function approximation problem [8]. In this model, the input is based on quantum bits (qubits). However, in many actual problems, the system input is the real vector in Hilbert space, and the method is not presented to transform the real to the quantum state in Ref. [8]. For the algorithm of model, the only two iterative equations in complex number are given based on the gradient descent algorithm. Neither the gradient computation formula nor the input-output relationship of networks is presented. Therefore, it is not easy for the reader to simulate the algorithm and apply this model to actual problem.

In this paper, based on the universality of the single qubit rotation gate and the two-qubit controlled-NOT gate, a quantum neuron model and three layers quantum back-propagation neural networks (QBP) model are proposed. The learning algorithm in Ref. [8] is improved. A transform method from the real to the qubit is proposed. On the basis of the probability amplitude vector of qubits, the input-output relationship of the model is presented. To facilitate the practical application, the learning algorithm is deducted in detail and the implementing scheme is given. The continuity of this model is theoretically proved. Two application examples are designed, and the simulation results show that this model and algorithm are evidently superior to the conventional back-propagation networks (CBP) in three aspects: convergence speed, convergence rate, and robustness.

\section{The Qubit and Quantum Gates}

\subsection{The Qubit}

In the quantum computation, the term "qubit" is introduced as the counterpart of the "bit" in the conventional computation to describe the state of quantum computation. In quantum computation system two quantum physical states labeled as $|0\rangle$ and $|1\rangle$ express 1 bit information. $|0\rangle$ corresponds to the bit 0 of classical computation, while the $|1\rangle$ to the bit 1 . The qubit state $|\phi\rangle$ maintains a coherent superposition of states $|0\rangle$ and $|1\rangle$

$$
|\phi\rangle=\alpha|0\rangle+\beta|1\rangle
$$

where $\alpha$ and $\beta$ are complex numbers called probability amplitude. That is, the qubit state $|\phi\rangle$ collapses into either $|0\rangle$ state with probability $|\alpha|^{2}$, or $|1\rangle$ state with probability $|\beta|^{2}$, and

$$
|\alpha|^{2}+|\beta|^{2}=1
$$

Therefore, the qubit can also be described by the probability amplitudes as $[\alpha, \beta]^{\mathrm{T}}$.

\subsection{The Quantum Gate}

The definition of single qubit rotation gate is described as follows

$$
R(\theta)=\left[\begin{array}{cc}
\cos \theta & -\sin \theta \\
\sin \theta & \cos \theta
\end{array}\right]
$$

Let the quantum state $|\phi\rangle=\left[\begin{array}{c}\cos \theta_{0} \\ \sin \theta_{0}\end{array}\right]$. Then the $|\phi\rangle$ can be evolved by $R(\theta)$ as follows $R(\theta)|\phi\rangle=\left[\begin{array}{c}\cos \left(\theta_{0}+\theta\right) \\ \sin \left(\theta_{0}+\theta\right)\end{array}\right]$. It is obvious that the $R(\theta)$ performs the phase rotation. 
The effect of the quantum NOT gate is exchange the two probability amplitudes of a qubit, and its definition is described as follows

$$
U=\left[\begin{array}{ll}
0 & 1 \\
1 & 0
\end{array}\right]
$$

Using the method proposed in Ref. [8], the controlled-NON gate can be realized by Equation (5)

$$
C(k)=\left[\begin{array}{cc}
\cos \left(\frac{\pi}{2} k-2 \theta_{0}\right) & -\sin \left(\frac{\pi}{2} k-2 \theta_{0}\right) \\
\sin \left(\frac{\pi}{2} k-2 \theta_{0}\right) & \cos \left(\frac{\pi}{2} k-2 \theta_{0}\right)
\end{array}\right]
$$

According to the different value of the controlled parameter $k$, the controlled effect includes three cases as follows

(1) If $k=1$, then $C(k)|\phi\rangle=\left[\begin{array}{c}\sin \theta_{0} \\ \cos \theta_{0}\end{array}\right]$, which corresponds to reversal rotation;

(2) If $k=0$, then $C(k)|\phi\rangle=\left[\begin{array}{c}\cos \theta_{0} \\ -\sin \theta_{0}\end{array}\right]$, which corresponds to not rotation. In this case, the phase of the probability amplitude of quantum state $|1\rangle$ is reversed. However, its observed probability is invariant so that we are able to regard this case as no-rotation;

(3) If $0<k<1$, then

$$
C(k)|\phi\rangle=\left[\begin{array}{c}
\cos \left(\frac{\pi}{2} k-\theta_{0}\right) \\
\sin \left(\frac{\pi}{2} k-\theta_{0}\right)
\end{array}\right]
$$

\section{The Quantum-Inspired Neural Networks Model}

\subsection{The Quantum-Inspired Neuron}

On the basis of the universal quantum gates, the quantum neuron model proposed in this paper includes five parts: input, phase rotation, aggregation, reverse rotation and output, where the input is described by qubits, the output is given by the probability of the state in which $|1\rangle$ is observed, the phase rotation and the reverse rotation are performed by quantum rotation gate $R\left(\theta_{i}\right)$ and controlled-NOT gate $U(\gamma)$, respectively. The quantum neuron model is shown in Figure 1.

Where the definition of $R\left(\theta_{i}\right)$ is same as Equation (3). The $U(\gamma)$ is defined as follows

$$
U(\gamma)=C(f(\gamma))
$$

where $f$ is a sigmoid function, and the definition of $C(\bullet)$ refers to Equation (5). Let $\left|x_{i}\right\rangle=\left[\begin{array}{ll}\cos t_{i} & \sin t_{i}\end{array}\right]^{\mathrm{T}}$, and $\theta=\arg \left(\sum_{i=1}^{n} R\left(\theta_{i}\right)\left|x_{i}\right\rangle\right)=\arctan \left(\sum_{i=1}^{n} \sin \left(t_{i}+\theta_{i}\right) / \sum_{i=1}^{n} \cos \left(t_{i}+\theta_{i}\right)\right)$. The result of aggregation is defined as

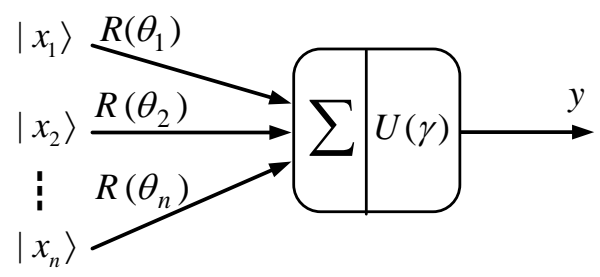

Figure 1. The quantum-inspired neuron model. 
follows

$$
\sum_{i=1}^{n} R\left(\theta_{i}\right)\left|x_{i}\right\rangle=\left[\begin{array}{ll}
\cos \theta & \sin \theta
\end{array}\right]^{\mathrm{T}}
$$

The result of reverse rotation is given by

$$
U(\gamma) \sum_{i=1}^{n} R\left(\theta_{i}\right)\left|x_{i}\right\rangle=\left[\cos \left(\frac{\pi}{2} f(\gamma)-\theta\right) \sin \left(\frac{\pi}{2} f(\gamma)-\theta\right)\right]^{\mathrm{T}}
$$

The input-output relationship of quantum neuron is described as follows

$$
y=\sin \left(\frac{\pi}{2} f(\gamma)-\theta\right)=\sin \left(\frac{\pi}{2} f(\gamma)-\arg \left(\sum_{i=1}^{n} R\left(\theta_{i}\right)\left|x_{i}\right\rangle\right)\right)
$$

\subsection{The Quantum-Inspired Neural Networks}

The quantum neural networks are composed of some quantum neurons and conventional neurons according to the definite linking rule. The three layers feed-forward QBP model proposed in this paper is shown in Figure 2, where the input layer and the hidden layer has $n, p$ quantum neurons, respectively, and the output layer has $m$ conventional neurons.

Suppose $\left|x_{i}\right\rangle$ are the networks input, $h_{j}$ are the hide layer output, $y_{k}$ are the networks output, $R\left(\theta_{i j}\right)$ are the quantum rotation gates to update qubits in the hide layer, $w_{j k}$ are the link-weights between the hide layer and the output layer, $C(0)$ and $U\left(\alpha_{j}\right)$ are the controlled-NOT gates that are regarded as the transformation function of quantum neurons in the input layer and hide layer, respectively. The input-output relationship of each layer is described as follows

$$
\begin{gathered}
h_{j}=\sin \left(\frac{\pi}{2} f\left(\alpha_{j}\right)-\arg \left(\sum_{i=1}^{n} R\left(\theta_{i j}\right)\left|x_{i}\right\rangle\right)\right) \\
y_{k}=g\left(\sum_{j=1}^{p} w_{j k} h_{j}\right)=g\left(\sum_{j=1}^{p} w_{j k} \sin \left(\frac{\pi}{2} f\left(\alpha_{j}\right)-\arg \left(\sum_{i=1}^{n} R\left(\theta_{i j}\right)\left|x_{i}\right\rangle\right)\right)\right)
\end{gathered}
$$

where $i=1,2, \cdots, n ; j=1,2, \cdots, p ; k=1,2, \cdots, m$.

\section{Training Algorithm}

For the training samples described in the $n$-dimension Euclid-space $\bar{X}=\left(\bar{x}_{1}, \bar{x}_{2}, \cdots, \bar{x}_{n}\right)^{\mathrm{T}}$, the transform formula to realize quantum state description of the training samples is defined as follows

$$
|X\rangle=\left[\left|x_{1}\right\rangle,\left|x_{2}\right\rangle, \cdots,\left|x_{n}\right\rangle\right]^{\mathrm{T}}
$$

where $\left|x_{i}\right\rangle=\cos \left(\frac{2 \pi}{1+\exp \left(-\bar{x}_{i}\right)}\right)|0\rangle+\sin \left(\frac{2 \pi}{1+\exp \left(-\bar{x}_{i}\right)}\right)|1\rangle=\left[\cos \left(\frac{2 \pi}{1+\exp \left(-\bar{x}_{i}\right)}\right) \sin \left(\frac{2 \pi}{1+\exp \left(-\bar{x}_{i}\right)}\right)\right]^{\mathrm{T}}$.

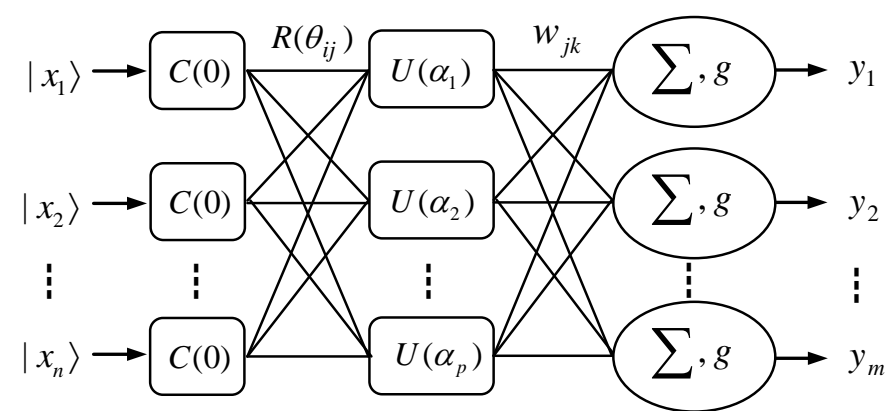

Figure 2. Three layers quantum-inspired networks model. 
In the QBP described by Figure 2, there are three groups of parameters, the rotation parameter $\theta_{i j}$, the reversal parameter $\alpha_{j}$ and the link-weights $w_{j k}$, that need updating. The error function is defined as follows

$$
E=\frac{1}{2}(\tilde{Y}-Y)^{2}
$$

where $\tilde{Y}$ and $Y$ are the normalized desired output and the practical output, respectively.

$$
\begin{gathered}
\text { Let }\left|x_{i}\right\rangle=\left[\begin{array}{c}
\cos \varphi_{i} \\
\sin \varphi_{i}
\end{array}\right], \quad \beta_{j}=\arctan \left(\frac{\sum_{i=1}^{n} \sin \left(\varphi_{i}+\theta_{i j}\right)}{\sum_{i=1}^{n} \cos \left(\varphi_{i}+\theta_{i j}\right)}\right) \text {, Equation (12) can be rewritten as } \\
y_{k}=g\left(\sum_{j=1}^{p} w_{j k} \sin \left(\frac{\pi}{2} f\left(\alpha_{j}\right)-\beta_{j}\right)\right)
\end{gathered}
$$

Let $S_{j}=\frac{\sum_{i=1}^{n} \sin \left(\varphi_{i}+\theta_{i j}\right)}{\sum_{i=1}^{n} \cos \left(\varphi_{i}+\theta_{i j}\right)}, \quad S_{j 1}=\sum_{i=1}^{n} \cos \left(\varphi_{i}+\theta_{i j}\right), \quad T_{j}=\frac{\cos \left(\varphi_{i}+\theta_{i j}\right) S_{j 1}+\sin ^{2}\left(\varphi_{i}+\theta_{i j}\right)}{S_{j 1}^{2}}$. According to the gradient descent algorithm,

$$
\begin{gathered}
\Delta \theta_{i j}=-\frac{\partial E}{\partial \theta_{i j}}=-\sum_{k=1}^{m}\left(\tilde{y}_{k}-y_{k}\right) g^{\prime} w_{j k} \cos \left(\frac{\pi}{2} f\left(\alpha_{j}\right)-\beta_{j}\right) \frac{T_{j}}{1+S_{j}^{2}} \\
\Delta \alpha_{j}=-\frac{\partial E}{\partial \alpha_{j}}=\frac{\pi}{2} \sum_{k=1}^{m}\left(\tilde{y}_{k}-y_{k}\right) g^{\prime} w_{j k} \cos \left(\frac{\pi}{2} f\left(\alpha_{j}\right)-\beta_{j}\right) f^{\prime} \\
\Delta w_{j k}=-\frac{\partial E}{\partial w_{j k}}=\left(\tilde{y}_{k}-y_{k}\right) g^{\prime} \sin \left(\frac{\pi}{2} f\left(\alpha_{j}\right)-\beta_{j}\right)
\end{gathered}
$$

The parameter updating rules are as follows

$$
\begin{gathered}
\theta_{i j}(t+1)=\theta_{i j}(t)+\eta \Delta \theta_{i j}(t) \\
\alpha_{j}(t+1)=\alpha_{j}(t)+\eta \Delta \alpha_{j}(t) \\
w_{j k}(t+1)=w_{j k}(t)+\eta \Delta w_{j k}(t)
\end{gathered}
$$

where $\eta$ is the learning coefficient.

\section{Simulation Comparisons}

To testify the validity of QBP, an actual example is designed and the QBP is compared with the CBP in this section. To make comparison equitable, the QBP adopts the same structure and parameters as CBP in the simulations.

The XOR problem has been the typical example of the neural networks learning algorithm research. This problem is the simplest example for nonlinear classification problem, which the corresponding optimization surface is irregular, and exists some local minimum. In this simulation, The XOR problem is generalized from four points to sixteen points. The point set is shown in Figure 3.

Firstly, we investigate how to change for the convergence rate when the learning coefficient changes. The networks structure is set to $2-10-1$, the restriction error is 0.1 , and the restriction iteration steps are 2000 . The learning coefficient is from $\{0.1,0.2, \cdots, 1.0\}$. This example is simulated 100 times for each learning coefficient by the QBP and the CBP, respectively. When the learning coefficient changes, the maximum of convergence rate of QBP is $87 \%$ and the minimum is $51 \%$. However, the convergence rate of CBP changes in a large range when the learning coefficient changes. When the learning coefficient is less than 0.2 , the convergence rate of CBP is $0 \%$, and when the learning coefficient is more than 0.2 , the maximum of convergence rate of CBP is 


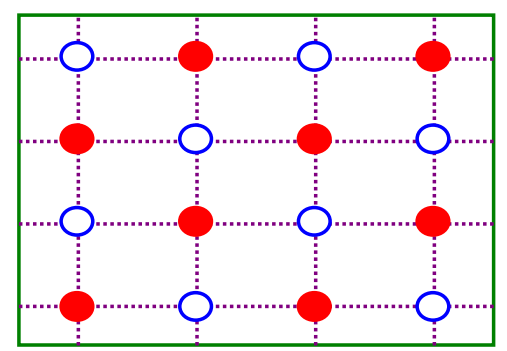

Figure 3. Sixteen points distribution on the plane.

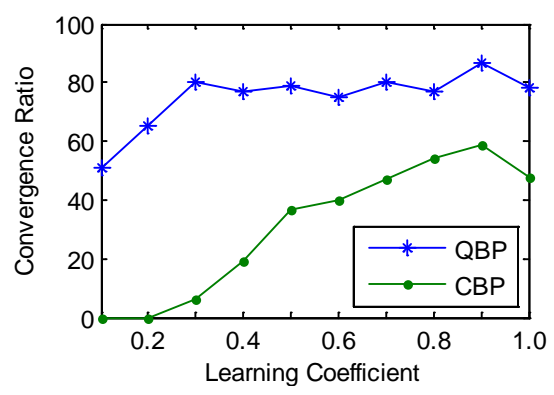

Figure 4. The relation of the convergence rate and $\eta$.

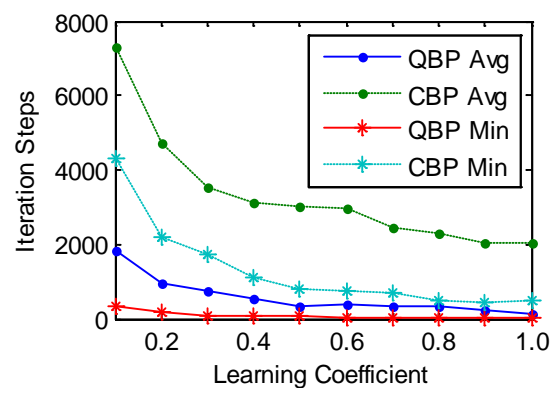

Figure 5. The relation of the iteration steps and $\eta$.

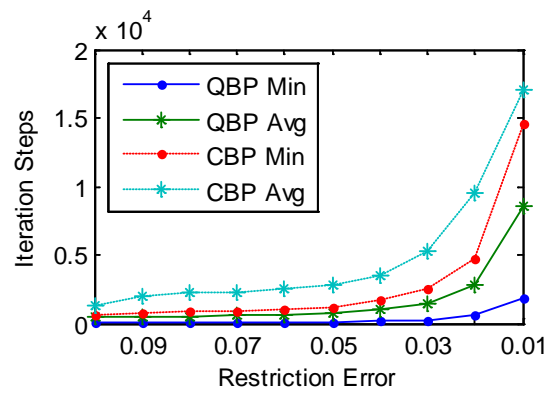

Figure 6. The relation between the iteration steps and the restriction error.

only 59\%. The comparison result is shown in Figure 4.

The comparison result shows that the QBP is evidently superior to the CBP in both the convergence rate and the robustness.

Secondly, we investigate how to change for the iteration steps when the learning coefficient changes. The networks structure is set to $2-10-1$, the restriction error is 0.1 , and the restriction iteration steps are 10,000 . The learning coefficient is still from $\{0.1,0.2, \cdots, 1.0\}$. This example is simulated 100 times for each learning coefficient by the QBP and the CBP, respectively. When the learning coefficient changes, for the average iteration steps of QBP, the maximum is 1838 steps, and the minimum is 151 steps; for the minimum iteration steps of 
QBP, the maximum is 336 steps, and the minimum is 27 steps. However, for the average iteration steps of CBP, the maximum is 7308 steps, and the minimum is 2021 steps; for the minimum iteration steps of CBP, the maximum is 4314 steps, and the minimum is 463 steps. The comparison result is shown in Figure 5.

The comparison result shows that the QBP is evidently superior to the CBP in both the iteration steps and its fluctuation range when the learning coefficient changes.

Finally, we investigate how to change for the iteration steps when the restriction error changes. The networks structure is set to 2-10-1. It is known from Figure 4 and Figure 5 that the performance of QBP and CBP is the best when the learning coefficient is set 0.9 . Therefore, the learning coefficient is set 0.9 . The restriction error is from $\{0.10,0.09, \cdots, 0.01\}$. This example is simulated 100 times for each restriction error by the QBP and the CBP, respectively. The comparison result is shown in Figure 6.

When the restriction error changes, for the average iteration steps of QBP, the maximum is 8575 steps, and the minimum is 431 steps; for the minimum iteration steps of QBP, the maximum is 1816 steps, and the minimum is 30 steps. However, for the average iteration steps of CBP, the maximum is 17,100 steps, and the minimum is 1304 steps; for the minimum iteration steps of CBP, the maximum is 14,525 steps, and the minimum is 659 steps. Hence, when the restriction error changes, the iteration steps and its fluctuation range of QBP is far less than that of CBP, which takes on the preferable robustness.

\section{Conclusion}

On the basis of the qubits and the universal quantum gates, a quantum BP neural networks model is proposed; the learning algorithm of this model is designed; and the continuity of this model is proved. The simulation result shows that this model and algorithm are superior to conventional BP networks in three aspects: the convergence speed, convergence rate, and robustness, by an actual example of pattern recognition and function approximation.

\section{References}

[1] Shor, P.W. (1994) Algorithms for Quantum Computation: Discrete Logarithms and Factoring. 35th Annual Symposium on Foundations of Computer Science, New Mexico, 20-22 November 1994, 124-134. http://dx.doi.org/10.1109/SFCS.1994.365700

[2] Grover, L.K. (1996) A Fast Quantum Mechanical Algorithm for Database Search. Proceedings of the 28th Annual ACM Symposium on the Theory of Computing, Pennsylvania, 212-221. http://dx.doi.org/10.1145/237814.237866

[3] Perus, M. (1996) Neuro-Quantum Parallelism in Brain-Mind and Computers. Informatics, 20, 173-183.

[4] Adenilton, J., Wilson, R. and Teresa, B. (2012) Classical and Superposed Learning for Quantum Weightless Neural Network. Neurocomputing, 75, 52-60. http://dx.doi.org/10.1016/j.neucom.2011.03.055

[5] Israel, G.C., Angel, G.C. and Belen, R.M. (2011) Dealing with Limited Data in Ballistic Impact Scenarios: An Empirical Comparison of Different Neural Network Approaches. Application Intelligence, 35, 89-109. http://dx.doi.org/10.1007/s10489-009-0205-8

[6] Kak, S. (1995) On Quantum Neural Computing. Information Sciences, 19, 143-160. http://dx.doi.org/10.1016/0020-0255(94)00095-S

[7] Zhu, D.Q. and Sang, Q.B. (2006) A Fault Diagnosis Algorithm for the Photovoltaic Radar Electronic Equipment Based on Quantum Neural Networks. Acta Electronica Sinica, 34, 573-576.

[8] Matsui, N., Kouda, N. and Nishimura, H. (2000) Neural Networks Based on QBP and Its Performance. Proceedings of the IEEE-INNS-ENNS International Joint Conference on Neural Networks, 3, 247-252.

http://dx.doi.org/10.1109/ijcnn.2000.861311 